Journal of Animal and Veterinary Advances 11 (7): 946-954, 2012

ISSN: $1680-5593$

(C) Medwell Journals, 2012

\title{
Correction of Angular Deformities with Ilizarov External Fixator in Dogs
}

\author{
Zihni Mutlu, Kemal Altunatmaz, Dilek Olgun Erdikmen, Yalcin Devecioglu and Ozlem Guzel \\ Department of Surgery, Faculty of Veterinary Medicine, University of Istanbul, \\ Avcilar, 34320 Istanbul, Turkey
}

\begin{abstract}
In this study, ten dogs with different breed, ages and genders were used for treathment of angular deformities. The limb deformities were discovered from the mediolateral and craniocaulal images of the preoperative radiographs. Apex of the deformity and the deformity plane were determined using graphical methods. Following ulnar ostectomy, rings were fixed to the distal and proximal segment of radius in all cases. After fixation of the wires to the ring radial osteotomy was performed. Deformations were corrected immediately in two case and progressively in eight cases. Latency period ranged from 3-7 days (in immature dogs 3-5 days, mature dogs 5-7 days). A distraction rate of $1 \mathrm{~mm} \mathrm{day}^{-1}$ was used in all dogs. Distraction was performed twice daily in all dogs. Minor wire track inflamation occured in eight dogs with three dogs also having wire track infection. No post-operative neurological impairment was observed in any dogs. Follow-up evalution by clinical and radiological examination ranged from 95-150 days. Limb functions were assessed to be excellent in four dogs, good in four dogs and fair in two dogs.
\end{abstract}

$\underline{\text { Key words: Angular deformity, ilizarov external fixator, dogs, neurological impairment, wire track, Turkey }}$

\section{INTRODUCTION}

The development and application of Circular External Skeletal Fixator (CESF) systems and the extensive utilization of the phenomenon of distraction osteogenesis by the Russian physician, Gavriil Abramovich Ilizarov have initiated a new orthopaedic treatment modality. These modular systems can be assembled in numerous configurations to perform bone lengthening and correction of angular, translational and rotational limb deformities (Fox et al., 1995; Lesser, 1994; Lewis et al., 1999b; Marcellin-Little, 1999; Stallings et al., 1998). With the Ilizarov external fixation method, a deformity can be corrected gradualy (with distraction osteogenesis) or in one stage (Latte, 2002; Preston, 2000). Limb deformities in dogs are seen most frequently in the antebrahium area (Latte, 1998; Marcellin-Little, 1999). Approximately, $0.74 \%$ of all bone problems in dogs are deformities found in the antebrachium area (Marcellin-Little, 1999). Radius and ulna grows together as a whole with carpal joints. Antebrachium's normal development depends on the synchronize growth of these bones. One of the most important causes of deformities observed in dogs front legs is due to unsynchronised enlargement in the radius and ulna after premature closure of the growth plates. The Distal Ulnar Growth Plate (DUGP) and the Distal Radial Growth Plate (DRGP) are the most affected. DUGP's slow growth or premature closure which results in an abnormally shortened ulna, restricts the normal longitudinal growth of the radius. This causes abnormal development of front legs syndrome known as radius curvus. In such cases, radius curvus, front limb shortening, carpal valgus (DUGP influencing), carpal varus (DRGP influencing), incongruity of elbow and wrist joints, lameness and in the future degenerative osteoarthritis are observed (Forell and Schwarz, 1993; Marcellin-Little et al., 1998; MarcellinLittle, 1999; Quinn et al., 2000; Stallings et al., 1998). Salter-Harris type V fractures, metabolic diseases, secondary trauma, chondrodysplasia, Hypertrophic osteodystrophy, Rickets, malnutrition, Intramedullary Pin applications affecting the growth plates are reasons that prevent the synchronized growth of the radius and ulna bones (Forell and Schwarz, 1993; Marcellin-Little et al., 1998; Quinn et al., 2000). In addition, angular deformities may occur as a result of conservative treatment of fractures and malunion of the bones (Ferretti, 1998). The aim of a deformity correction is to make the aligment of the foot normal and to ensure the normal orientation of each joints mechanical axis. The same level of deformity seen in both craniocaudal and mediolateral radiographs is called the oblique plan deformity (Marcellin-Little et al., 1998). The most ideal method of correcting the oblique plan deformity is gradual correction with the use of a motor and hinged fixator. Deformity correction process should not be considered only as a correction of bone tissue. During this process, the positions and sizes of the muscles, tendons, veins and nerves changes. Vascular and nerve tissue can tolerate $1 \mathrm{~mm}$ growth in $24 \mathrm{~h}$. The best bone regeneration has been reported to be daily

Corresponding Author: Zihni Mutlu, Department of Surgery, Faculty of Veterinary Medicine, University of Istanbul, Avcilar, 34320 Istanbul, Turkey 
$4 \times 0.25 \mathrm{~mm}$ growth. But muscle tissue has been seen to be more resistant to growth and even with a $4 \times 0.25 \mathrm{~mm}$ growth, contracture of the muscles occures (Ilizarov, 1989a; Marcellin-Little et al., 1998; Stallings et al., 1998; Yanoff et al., 1992). The purpose of this study is to show the successful results of deformity corrections done with Ilizarov External Fixator System (IEFS).

\section{MATERIALS AND METHODS}

The material of the study was made up of ten dogs of different breed, ages and genders brought to the Department of Surgery with complaints of curvatures in the front legs as a result of oblique plan deformity in their antebrachium which was determined after clinical and radiological examinations. As fixation materials researchers used, 100-120-130 mm diameter full, half and $5 / 8$ rings from the Ilizarov set, $3 \mathrm{~mm}$ diameter Schanz screws and holders to suit them, 1.6-1.8 mm Kirschner pins and hinges. The limb deformities were discovered from the mediolateral and craniocaulal images of the preoperative radiographs. Apex of the deformity (proximal-to-distal) and the deformity plane (circumferential) were determined using graphical methods (Fig. 1a-d) (Lewis et al., 1999b). Both the radius and ulna lengths of the front legs were also recorded. Rotational deformity of the elbow and carpal joints were calculated by comparing the levels of flexion/extension during the physical examination. Measurement of limb deformities were performed over the radiograph.

In cases where the level of deformity is diaphyseal, two rings were determined at the proximal and distal

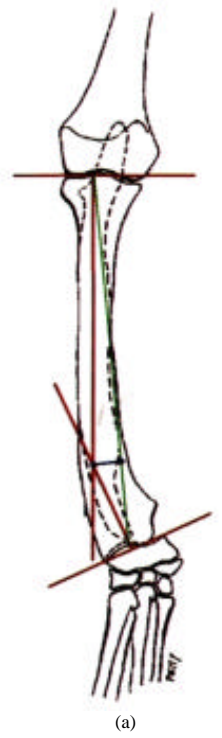

(a)

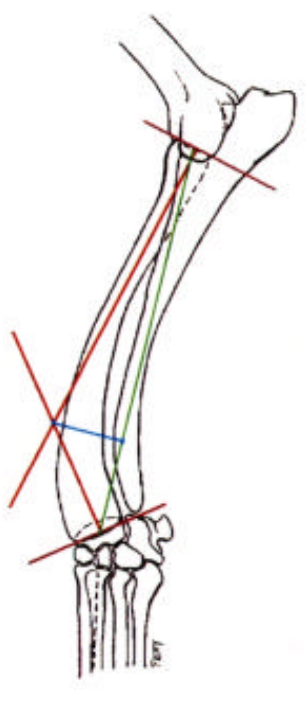

(b)

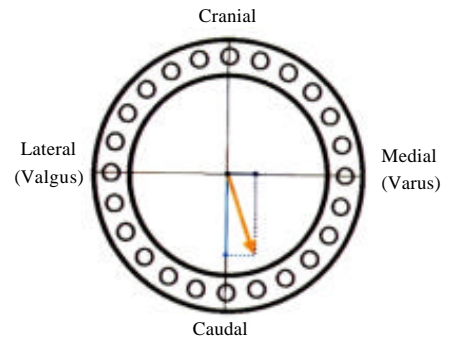

(c)

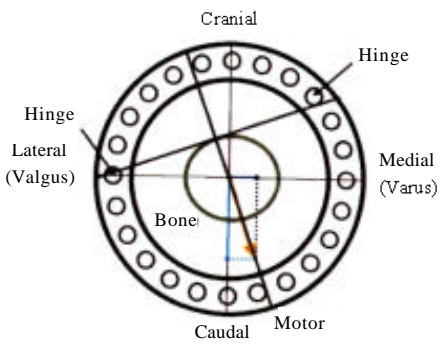

(d)

Fig. 1: A schematic drawing of the radiographic image of an antebrachium deformity in a dog; a) craniocaudal and b) mediolateral. Two parallel lines (purple lines) were drawn to the neighboring joints (art.cubiti and art.carpi). Two more lines are drawn (red lines) which are perpendicular to the joint faces and divide the metaphyseal portions of the radius in two. These two lines (red lines) determined the intersection point of the deformity apex (CORA-Center of Rotation of angulation). From the craniocaudal radiography, the angles of the valgus/varus and from the lateral radiography the angles of the procurvatum were calculated. The valgus/varus and craniocaudal components of the deformity were calculated from their respective orthogonal view radiograph: A line was then drawn connecting the center of the articular surface of the proximal and distal radius (green line). Another line was drawn perpendicular to the line bisecting the center of the articular surfaces and passing through the previously defined apex of the deformity. The measured length of this line (blue line) on the craniocaudal view radiograph constituted the varus or valgus component (vector) of the deformity. The measured length of this line (turquoise line) on the lateral view radiographically constituted the cranial or caudal component (vector) of the deformity; c) If the deformity was in an oblique plan (not only in the craniocaudal or mediolateral plane) the plane of deformity was determined by plotting the two vector components of the deformity on an $\mathrm{x}$ (varus/valgus)/y (craniocaudal) grid. The resultant vector (orange line) of these two components defined the plane of the deformity; d) Hingeds were placed at the apex of the deformity. A line drawn between hinges was tangential to the convex cortex of the bone and perpendicular to the plane of deformity. The angular motor was placed on the side of the ring opposide the hinges in or parallel to the plane of deformity 
fragments of the bone. In the cases where the level of deformity is metaphyseal, 1 ring was used in a small fragment and 2 rings were used in large fragmented. In addition to the Kirshner wires used in the ring on a small fragment, a $3 \mathrm{~mm}$ diameter Schanz screw was also used. In the two cases with elbow dysplasia (No. 2 and 6) a proximal ulna osteotomy was performed. Using IEF, angular corrections were made and hinges were placed between the ring blocks. Ulnar osteotomy or ostectomisi was performed and IEF was applied. As determined from the preoperative radiographs hinges were placed in the apex of the deformity. Hinges were placed to a line perpendicular to the deformity plan and tangent to the radius of the convex cortex. The angular motor was placed in the deformity plane or paralel to it. After all the pins were put through the rings, radial osteotomy was preformd on the deformity apex or just under it.

Distraction protocols were defined by the latency period, rate, rhythm, duration of distraction period and amount of lenghtening performed. Latency is the time (3-7 days) from surgical application of the fixator to initiating distraction. The rate is the total amount ( $\mathrm{mm}$ ) of distraction performed each day which was measured along the concave cortex of the radius. The rhythm is the number of increments of distraction performed each day. The total amount that was stretched was measured and recorded in mm as per radiograph. By the use of a Circular Fixator (CF) with an angular motor, to create a distraction of $1 \mathrm{~mm}$ a day of the bone tissue deformity corrections was done by taking advantage of the similar triangles method in geometry (Lesser, 1998; Marcellin-Little et al., 1998) the distraction ratio was calculated. About 3-7 days after the surgery, the frequency of distraction was preformed twice a day $(2 \times 0.5 \mathrm{~mm})$ in the mornings and evenings until the angle of deformity is redolved. In patients with deformity and limb shortness after adjusting the angle of deformity with distraction osteogenesis, the motor unit and the hinges are removed, $2 \times 0.5 \mathrm{~mm}$ distraction was performed on the radius using rods. When the short radius reaches its the normal size (the size of the radius on the opposite side) the implementation of distraction is cesed. In one case (No. 5), deformity was corrected in a single-stage and distraction was performed with linear motors.

After the operation by revising the entire system, neurological, vascular and muskular structures were viewed to see whether there was activity. It was suggested to the owners of the patients that the pin bottoms be cleaned daily with $10 \%$ povidone iode. Complications were recorded during the convalescent period. During the clinical and radiological controls, the postoperative presence of neural and vascular disorders, the functionality of the fixation applied distal and proximal joints of the bones, pin-track infections, tendon and muscle contractures, pain and wether they could use extremities were evaluated. The wire truck inflammation which was controled by local administration of antibiotic therapy were assessed as minor complications. The wire truck inflammations and infections which could not be controlled by local application of antibiotic treatment were classified as major complications (Lewis et al., 1999a). Using the radiographs taken, the consolidation of the bone tissue and the degree of callus formation between the fragments were examined. After consolidation was completed the fixator was removed by applying sedation. Long-term follow-up evaluations of the animals was achieved by direct examination. Foot functions were graded as the following scale (Forell and Schwarz, 1993; Fox et al., 1995) excellent: a normal limb function; good; slight lameness only after extensive exercise; fair: slight to moderate lameness but consistent weight-bearing and poor: non-weight-bearing lameness. Cosmetic appearance was also assessed (Forell and Schwarz, 1993; Fox et al., 1995) excellent. The same appearance of the opposite side normal leg; good; only a small difference when compared to the opposite leg, fair; a significant difference in appearance when compared against the opposite leg; poor; marked and disfigured alteration in appearance compared to the contralateral limb.

\section{RESULTS}

Deformity correction were performed in all dogs on one leg (Table 1 and 2). The ages of the dogs ranged from 7-18 months and body weights ranged from $13-38 \mathrm{~kg}$. In 9 of the cases, the oblique plan deformity and in one case (No. 5) frontal plane deformity was viewed. Two dogs (No. 5 and 10) underwent linear antebrachial lengthening. Dog No. 10 had antebrachial (oblique plan) deformity secondary to premature distal ulnar phiseal closure (Fig. 2). Distal diaphyseal osteotomy and periosteal excision of the radius was done after distal diaphyseal ostectomy of the ulna. The dog's valgus and rotational correction was corrected acutely. Linear distraction in dog (No. 10) was performed for 29 days resulting in $25 \mathrm{~mm}$ increase in radial lenght. Distal radial segment was distracted to lengthen the antebrachium. The radius was lenghtened to contralateral normal radial lenght in this dog. This dog has occasional lameness following periods of extensive exercise or prolonged rest and an good cosmetic result 145 days following the operation. 
Table 1: Clinical summaries of $\operatorname{dogs}(n=10)$ undergoing correction of angular, rotational and length deformities using the Ilizarov External Skelet Fixator System

\begin{tabular}{|c|c|c|c|c|c|c|c|}
\hline $\begin{array}{l}\text { Case } \\
\text { no. }\end{array}$ & Signalment & Description of deformity & Procedure & Fixator construct & $\begin{array}{l}\text { Distraction protocol } \\
\text { (Day after operation) }\end{array}$ & Complications & $\begin{array}{l}\text { Final functional and } \\
\text { cosmetic result }\end{array}$ \\
\hline 1 & $\begin{array}{l}20 \mathrm{~kg} \\
7 \text { months old } \\
\text { male pit bull }\end{array}$ & $\begin{array}{l}\text { Valgus, caudal and } \\
\text { angulation of the left } \\
\text { antebrachiun with } \\
\text { shortening secondary } \\
\text { to premature distal ulnar } \\
\text { physeal closure from } \\
\text { retained cartilage cores }\end{array}$ & $\begin{array}{l}\text { Left antebrachim: } \\
\text { distal ulnar } \\
\text { ostectomy, distal } \\
\text { radial osteotomy } \\
\text { and progresiv angular } \\
\text { correction of valgus }\end{array}$ & $\begin{array}{l}\text { Four } 100 \mathrm{~mm} \text { rings: } \\
\text { two rings proximal } \\
\text { and two rings distal } \\
\text { (with two wires). Hinges } \\
\text { and angular motor } \\
\text { between ring blocks }\end{array}$ & $\begin{array}{l}\text { Latency: } 3 \text { days } \\
\text { Rate }: 1 \mathrm{~mm} \\
\text { Rhytim: twice daily } \\
\text { Days distracted: } \\
15 \text { days; Lenthening: } \\
15 \mathrm{~mm} \text { at distal radial } \\
\text { osteotomy; Fixator } \\
\text { removal: } 53 \text { days }\end{array}$ & $\begin{array}{l}\text { Minor wire track } \\
\text { inflammation }\end{array}$ & $\begin{array}{l}\text { Good limb function } \\
\text { Excellent cosmetic } \\
\text { result ( } 95 \text { days) }\end{array}$ \\
\hline 2 & $\begin{array}{l}13 \mathrm{~kg} \\
36 \text { months } \\
\text { old female } \\
\text { crossbreed }\end{array}$ & $\begin{array}{l}\text { Valgus, caudal and } \\
\text { angulation of the left } \\
\text { antebrachiun with } \\
\text { shortening secondary } \\
\text { to premature distal ulnar } \\
\text { physeal closure due to } \\
\text { former ulnar fracture, } \\
\text { elbow incongruity }\end{array}$ & $\begin{array}{l}\text { Left antebrachim: } \\
\text { distal ulnar ostectomy, } \\
\text { distal radial osteotomy, } \\
\text { proximal ulnar osteotomy } \\
\text { and progresiv angular } \\
\text { correction of valgus }\end{array}$ & $\begin{array}{l}\text { Three } 100 \mathrm{~mm} \text { rings: } \\
\text { one ring proximal, } \\
\text { one ring diaphysial } \\
\text { and one ring distal } \\
\text { (with two wire) } \\
\text { The third ring supported } \\
\text { with one schanz wire } \\
\text { Hinges and angular } \\
\text { motor between second } \\
\text { and third rings }\end{array}$ & $\begin{array}{l}\text { Latency: } 7 \text { days } \\
\text { Rate: } 1 \mathrm{~mm} \\
\text { Rhytim: twice daily } \\
\text { Days distracted: } \\
17 \text { days; Lenthening: } \\
\text { Lenthening: } \\
17 \mathrm{~mm} \text { at distal radial } \\
\text { osteotomy } \\
\text { Fixator removal: } \\
90 \text { days }\end{array}$ & $\begin{array}{l}\text { Minor and major } \\
\text { wire track } \\
\text { inflammation } \\
\text { and infection. } \\
\text { Incompl ate } \\
\text { correction of } \\
\text { proximal radioulnar } \\
\text { subluxation. } \\
\text { Persistent mild valgus } \\
\text { deformity }\end{array}$ & $\begin{array}{l}\text { Fair limb function: } \\
\text { limited range of } \\
\text { motion in left elbow } \\
\text { Fair cosmetic } \\
\text { result (146 days) } \\
\text { us }\end{array}$ \\
\hline 3 & $\begin{array}{l}38 \mathrm{~kg} \\
8 \text { months old } \\
\text { male } \\
\text { Anatolian } \\
\text { shebperd dog }\end{array}$ & $\begin{array}{l}\text { Valgus, caudal and } \\
\text { external rotational } \\
\text { deformity of the right } \\
\text { antebrachiun with } \\
\text { shortening secondary } \\
\text { to premature distal } \\
\text { ulnar physeal closure } \\
\text { from retained cartilage } \\
\text { cores }\end{array}$ & $\begin{array}{l}\text { Right antebrachim: } \\
\text { distal ulnar ostectomy, } \\
\text { distal radial osteotomy } \\
\text { and acute rotational } \\
\text { correction and } \\
\text { progresiv angular } \\
\text { correction of valgus }\end{array}$ & $\begin{array}{l}\text { Four } 130 \mathrm{~mm} \text { rings: } \\
\text { two rings proximal } \\
\text { and two rings } \\
\text { distal (with two wires) } \\
\text { Hinges and } \\
\text { angular motor } \\
\text { between ring blocks }\end{array}$ & $\begin{array}{l}\text { Latency: } 5 \text { days } \\
\text { Rate }: 1 \mathrm{~mm} \\
\text { Rhytim: twice daily } \\
\text { Days distracted: } \\
23 \text { days } \\
\text { Lenthening: } 21 \mathrm{~mm} \\
\text { at distal radial } \\
\text { osteotomy Fixator } \\
\text { removal: } 93 \text { days }\end{array}$ & $\begin{array}{l}\text { Minor wire track } \\
\text { inflammation } \\
\text { temporary mild } \\
\text { flexure contracture }\end{array}$ & $\begin{array}{l}\text { Excell ent limb } \\
\text { function good } \\
\text { cosmetic result } \\
(150 \text { days })\end{array}$ \\
\hline 4 & $\begin{array}{l}32 \mathrm{~kg} \\
8 \text { months old } \\
\text { female } \\
\text { Anatolian } \\
\text { shebperd dog }\end{array}$ & $\begin{array}{l}\text { Valgus, caudal and } \\
\text { external rotational } \\
\text { deformity of the left } \\
\text { antebrachiun with } \\
\text { shortening secondary } \\
\text { to premature distal } \\
\text { ulnar physeal closure }\end{array}$ & $\begin{array}{l}\text { Left antebrachim: } \\
\text { distal ulnar ostectomy, } \\
\text { distal radial osteotomy } \\
\text { and acute rotational } \\
\text { correction and } \\
\text { progresiv angular } \\
\text { correction of valgus }\end{array}$ & $\begin{array}{l}\text { Four } 130 \mathrm{~mm} \text { rings: } \\
\text { two rings proximal } \\
\text { and two rings distal } \\
\text { (with two wires) } \\
\text { Hinges and angular } \\
\text { motor between } \\
\text { ring blocks }\end{array}$ & $\begin{array}{l}\text { Latency: } 5 \text { days } \\
\text { Rate }: 1 \mathrm{~mm} \\
\text { Rhytim: twice daily } \\
\text { Days distracted: } \\
23 \text { days } \\
\text { Lenthening: } 20 \mathrm{~mm} \\
\text { at distal radial } \\
\text { osteotomy } \\
\text { Fixator removal: } \\
88 \text { days }\end{array}$ & $\begin{array}{l}\text { Minor and major } \\
\text { wire track } \\
\text { inflammation } \\
\text { and infection } \\
\text { temporary } \\
\text { mild flexure } \\
\text { contracture }\end{array}$ & $\begin{array}{l}\text { Good limb function } \\
\text { good cosmetic } \\
\text { result } \\
\text { (145 days) }\end{array}$ \\
\hline 5 & $\begin{array}{l}31 \mathrm{~kg} \\
18 \text { months old } \\
\text { male } \\
\text { German } \\
\text { shebperd }\end{array}$ & $\begin{array}{l}\text { Left carpal } \\
\text { subluxation with } \\
\text { varus and cranial } \\
\text { angulation and } \\
\text { shortening } \\
\text { secondary to } \\
\text { eccentric premature } \\
\text { closure of the left } \\
\text { radius }\end{array}$ & $\begin{array}{l}\text { Left antebrachim: } \\
\text { distal ulnar ostectomy, } \\
\text { distal radial } \\
\text { osteotomy and } \\
\text { acute angular } \\
\text { correction of varus }\end{array}$ & $\begin{array}{l}\text { Three } 130 \mathrm{~mm} \text { rings: } \\
\text { one ring proximal, } \\
\text { one ring diaphysial } \\
\text { and one ring } \\
\text { distal (with two wire) } \\
\text { the third ring } \\
\text { supported with } \\
\text { one schanz wire } \\
\text { one schanz wire } \\
\text { secend and third rings }\end{array}$ & $\begin{array}{l}\text { Latency: } 7 \text { days } \\
\text { Rate }: 1 \mathrm{~mm} \\
\text { Rhytim: twice daily } \\
\text { Days distracted: } \\
10 \text { days } \\
\text { Lenthening: } 10 \mathrm{~mm} \\
\text { at distal radial } \\
\text { osteotomy } \\
\text { Fixator removal: } \\
58 \text { days }\end{array}$ & & $\begin{array}{l}\text { Excellentlimbfunction } \\
\text { excellent cosmetic } \\
\text { result } \\
\text { (138 days) }\end{array}$ \\
\hline 6 & $\begin{array}{l}21 \mathrm{~kg} \\
8 \text { months old } \\
\text { male } \\
\text { Setter }\end{array}$ & $\begin{array}{l}\text { Valgus, caudal } \\
\text { and external } \\
\text { rotational deformity } \\
\text { of the left } \\
\text { antebrachiun with } \\
\text { shortening } \\
\text { secondary to } \\
\text { premature distal } \\
\text { ulnar physeal closure } \\
\text { due to former radial } \\
\text { fracture, elbow } \\
\text { incongruity }\end{array}$ & $\begin{array}{l}\text { Left antebrachim: } \\
\text { Distal ulnar ostectomy, } \\
\text { distal radial } \\
\text { osteotomy, proximal } \\
\text { ulnar osteotomy } \\
\text { and acute rotational } \\
\text { correction and } \\
\text { progresive } \\
\text { angular correction } \\
\text { of valgus }\end{array}$ & $\begin{array}{l}\text { Three } 120 \mathrm{~mm} \text { rings: } \\
\text { One ring proximal, } \\
\text { one ring diaphysial } \\
\text { and one ring distal } \\
\text { (with two wire) } \\
\text { The third ring } \\
\text { supported with } \\
\text { one schanz wire } \\
\text { Hinges and angular } \\
\text { motor between } \\
\text { second and third rings }\end{array}$ & $\begin{array}{l}\text { Latency: } 3 \text { days } \\
\text { Rate: } 1 \mathrm{~mm} \\
\text { Rhytim: twice daily } \\
\text { Days distracted: } \\
19 \text { days } \\
\text { Lenthening: } 14 \mathrm{~mm} \\
\text { at distal radial } \\
\text { osteotomy } \\
\text { Fixator removal: } \\
45 \text { days }\end{array}$ & $\begin{array}{l}\text { Incomplate } \\
\text { correction } \\
\text { of proximal } \\
\text { radioulnar } \\
\text { subluxation }\end{array}$ & $\begin{array}{l}\text { Fair limb function: } \\
\text { limited range } \\
\text { of motion in } \\
\text { left elbow } \\
\text { good cosmetic } \\
\text { result } \\
(150 \text { days })\end{array}$ \\
\hline 7 & $\begin{array}{l}32 \mathrm{~kg} \\
10 \text { months old } \\
\text { male } \\
\text { Anatolian } \\
\text { shebperd dog }\end{array}$ & $\begin{array}{l}\text { Valgus deformity } \\
\text { of the rightt } \\
\text { antebrachiun with } \\
\text { shortening secondary } \\
\text { to premature } \\
\text { distal ulnar physeal } \\
\text { closure }\end{array}$ & $\begin{array}{l}\text { Right antebrachim: } \\
\text { Distal ulnar ostectomy, } \\
\text { distal radial } \\
\text { osteotomy and } \\
\text { progressive angular } \\
\text { correction of } \\
\text { valgus }\end{array}$ & $\begin{array}{l}\text { Four } 130 \mathrm{~mm} \text { rings: } \\
\text { two rings proximal } \\
\text { and two rings distal } \\
\text { (with two wires) } \\
\text { Hinges and } \\
\text { angular motor } \\
\text { between ring blocks }\end{array}$ & $\begin{array}{l}\text { Latency: } 5 \text { days } \\
\text { Rate }: 1 \mathrm{~mm} \\
\text { Rhytim: twice daily } \\
\text { Days distracted: } \\
13 \text { days } \\
\text { Lenthening: } 13 \mathrm{~mm} \\
\text { at distal radial } \\
\end{array}$ & $\begin{array}{l}\text { Minor wire track } \\
\text { inflammation }\end{array}$ & $\begin{array}{l}\text { Excellentlimbfunction } \\
\text { excellent cosmetic } \\
\text { result (115 days) }\end{array}$ \\
\hline
\end{tabular}




\begin{tabular}{|c|c|c|c|c|c|c|c|}
\hline $\begin{array}{l}\text { Case } \\
\text { No. }\end{array}$ & Signalment & Description of deformity & Procedure & Fixator construct & $\begin{array}{l}\text { Distraction protocol } \\
\text { (day after operation) }\end{array}$ & Complications & $\begin{array}{l}\text { Final functional and } \\
\text { cosmetic result }\end{array}$ \\
\hline 8 & $\begin{array}{l}28 \mathrm{~kg} \\
9 \text { months old } \\
\text { male German } \\
\text { shebperd }\end{array}$ & $\begin{array}{l}\text { Valgus deformity } \\
\text { of the left antebrachiun } \\
\text { with shortening } \\
\text { secondary to } \\
\text { premature distal } \\
\text { ulnar physeal } \\
\text { closure }\end{array}$ & $\begin{array}{l}\text { Left antebrachim: } \\
\text { distal ulnar } \\
\text { ostectomy, distal } \\
\text { radial osteotomy } \\
\text { and progressive } \\
\text { angular correction } \\
\text { of valgus }\end{array}$ & $\begin{array}{l}\text { Three } 120 \mathrm{~mm} \text { rings: } \\
\text { one ring proximal, } \\
\text { one ring diaphysial } \\
\text { and one ring distal } \\
\text { (with two wire) } \\
\text { the third ring } \\
\text { supported with } \\
\text { one schanz wire } \\
\text { Hinges and angular } \\
\text { motors between }\end{array}$ & $\begin{array}{l}\text { osteotomy } \\
\text { Fixator removal: } \\
50 \text { days } \\
\text { Latency: } 5 \text { days } \\
\text { Rate: } 1 \mathrm{~mm} \\
\text { Rhytim: twice daily } \\
\text { Days distracted: } \\
7 \text { days } \\
\text { Lenthening: } 7 \mathrm{~mm} \\
\text { at distal radial } \\
\text { osteotomy } \\
\text { Fixator removal: } \\
58 \text { days }\end{array}$ & $\begin{array}{l}\text { Minor wire track } \\
\text { inflammation }\end{array}$ & $\begin{array}{l}\text { Excellentlimbfunction } \\
\text { excellent cosmetic } \\
\text { result (135 days) }\end{array}$ \\
\hline 9 & $\begin{array}{l}27 \mathrm{~kg} \\
7 \text { months old } \\
\text { male } \\
\text { Anatolian } \\
\text { shebperd dog }\end{array}$ & $\begin{array}{l}\text { Valgus, caudal } \\
\text { and external } \\
\text { rotational deformity } \\
\text { of the right } \\
\text { antebrachiun } \\
\text { with shortening } \\
\text { secondary to } \\
\text { premature distal } \\
\text { ulnar physeal } \\
\text { closure from } \\
\text { retained cartilage } \\
\text { cores }\end{array}$ & $\begin{array}{l}\text { Right antebrachim: } \\
\text { distal ulnar ostectomy, } \\
\text { distal radial } \\
\text { osteotomy and } \\
\text { acute rotational } \\
\text { correction and } \\
\text { progresiv angular } \\
\text { correction of valgu }\end{array}$ & $\begin{array}{l}\text { Three } 120 \mathrm{~mm} \text { rings: } \\
\text { One ring proximal, } \\
\text { one ring diaphysial } \\
\text { and one ring distal } \\
\text { (with two wire) } \\
\text { the third ring } \\
\text { supported with } \\
\text { one schanz wire } \\
\text { Hinges and } \\
\text { angular motor between } \\
\text { second and third rings }\end{array}$ & $\begin{array}{l}\text { Latency: } 5 \text { days } \\
\text { Rate: } 1 \mathrm{~mm} \\
\text { Rhytim: twice daily } \\
\text { Days distracted: } \\
21 \text { days } \\
\text { Lenthening: } 20 \mathrm{~mm} \\
\text { at distal radial } \\
\text { osteotomy } \\
\text { Fixator removal: } \\
75 \text { days }\end{array}$ & $\begin{array}{l}\text { Minor and major } \\
\text { wire track } \\
\text { inflammation } \\
\text { and infection }\end{array}$ & $\begin{array}{l}\text { Good limb function } \\
\text { excellent cosmetic } \\
\text { result ( } 142 \text { days) }\end{array}$ \\
\hline 10 & $\begin{array}{l}24 \mathrm{~kg} \\
7 \text { months old } \\
\text { male } \\
\text { Anatolian } \\
\text { shebperd dog }\end{array}$ & $\begin{array}{l}\text { Valgus, caudal and } \\
\text { external rotational } \\
\text { deformity of the } \\
\text { right antebrachiun } \\
\text { with shortening } \\
\text { secondary to } \\
\text { premature distal } \\
\text { ulnar physeal } \\
\text { closure }\end{array}$ & $\begin{array}{l}\text { Right antebrachim: } \\
\text { distal ulnar ostectomy, } \\
\text { distal radial osteotomy } \\
\text { and acute rotational } \\
\text { correction and acute } \\
\text { angular correction } \\
\text { of valgu }\end{array}$ & $\begin{array}{l}\text { Four } 120 \mathrm{~mm} \text { rings: } \\
\text { two proximal and } \\
\text { two rings distal } \\
\text { (with two wires) } \\
\text { Linear motors } \\
\text { between ring } \\
\text { blocks }\end{array}$ & $\begin{array}{l}\text { Latency: } 3 \text { days } \\
\text { Rate: } 1 \mathrm{~mm} \\
\text { Rhytim: twice daily } \\
\text { Days distracted: } \\
29 \text { days } \\
\text { Lenthening: } 25 \mathrm{~mm} \\
\text { at distal radial } \\
\text { osteotomy } \\
\text { Fixator removal: } \\
83 \text { days }\end{array}$ & $\begin{array}{l}\text { Minor wire track } \\
\text { inflammation } \\
\text { Persistent mild } \\
\text { valgus deformity }\end{array}$ & $\begin{array}{l}\text { Good limb function } \\
\text { good cosmetic result } \\
\text { (145 days) }\end{array}$ \\
\hline
\end{tabular}

Table 2: Pre and postoperative data of dogs $(\mathrm{n}=10)$ undergoing limb deformity correction using the Ilizarov External Skelet Fixator System

\begin{tabular}{|c|c|c|c|c|}
\hline Case No. & $\begin{array}{l}\text { Preoperative craniocaudal carpal } \\
\text { valgus/varus (degrees) }\end{array}$ & $\begin{array}{l}\text { Preoperative mediolateral } \\
\text { radial angulation (degrees) }\end{array}$ & $\begin{array}{l}\text { Postoperative craniocaudal carpal } \\
\text { valgus/varus (degrees) }\end{array}$ & $\begin{array}{l}\text { Postoperative mediolateral } \\
\text { radial angulation (degrees) }\end{array}$ \\
\hline 1 & $21 \mathrm{vl}$ & $40 \mathrm{cu}$ & $17 \mathrm{vl}$ & $15 \mathrm{cu}$ \\
\hline 2 & $21 \mathrm{vl}$ & $23 \mathrm{cu}$ & $11 \mathrm{vl}$ & $9 \mathrm{cu}$ \\
\hline 3 & $25 \mathrm{vl}$ & $70 \mathrm{cu}$ & $12 \mathrm{vl}$ & $18 \mathrm{cu}$ \\
\hline 4 & $50 \mathrm{vl}$ & $45 \mathrm{cu}$ & $25 \mathrm{vl}$ & $13 \mathrm{cu}$ \\
\hline 5 & $30 \mathrm{vr}$ & 0 & $5 \mathrm{vr}$ & 0 \\
\hline 6 & $42 \mathrm{vl}$ & $40 \mathrm{cu}$ & $15 \mathrm{vl}$ & $14 \mathrm{cu}$ \\
\hline 7 & $25 \mathrm{vl}$ & $30 \mathrm{cu}$ & $9 \mathrm{vl}$ & $7 \mathrm{cu}$ \\
\hline 8 & $30 \mathrm{vl}$ & $28 \mathrm{cu}$ & $7 \mathrm{vl}$ & $7 \mathrm{cu}$ \\
\hline 9 & $23 \mathrm{vl}$ & $65 \mathrm{cu}$ & $12 \mathrm{vl}$ & $23 \mathrm{cu}$ \\
\hline 10 & $60 \mathrm{vl}$ & $70 \mathrm{cu}$ & $29 \mathrm{vl}$ & $18 \mathrm{cu}$ \\
\hline
\end{tabular}

vl: valgus; vr: varus; cu: caudal

Dog No. 5 with frontal plan deformity underwent a linear lenghtening following acute correction of a marked varus which was secondary to eccentric premature closure of the radius. Although, this dog was determined 30 degrees varus in preoperative craniocaudal radiograph, there was no pathological radial angulation in mediolateral radiograph. Linear distraction was performed for 10 days to increase radial lenght up to $10 \mathrm{~mm}$ which was calculated prior to the operation. Correrection of angular deformities (Fig. 3), utilizing paired hinges and an angular motor was performed in eight dogs (Dogs No. 1, 2, 3, 4, 6, 7, 8, 9). Correction of the rotational component of the deformity was performed acutely at the time of the operation in five dogs (Dog No. 3, 4, 6, 9 and 10). Proximal ulnar osteotomy was used in $\operatorname{dog}$ (No. 2 and 6) to resolve elbow incongruity. Although, proximal ulnar segments were distructed, the elbow incongruities were not resolved totaly.

Hinges and angular motors were repositioned in two dogs (No. 1 and 7) because the hinges were not 


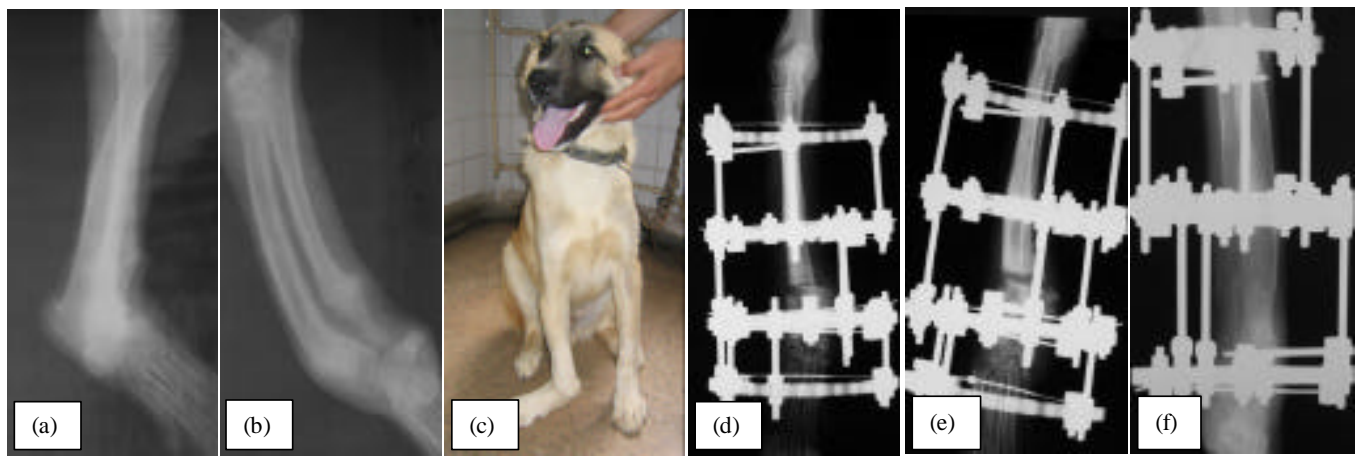

Fig. 2: a, b) Craniocaudal and lateral view radiographs of the right antebrachium of dog (No. 10). The distal ulnar physis has closed prematurely; c) View of the dog befor operation with carpal valgus and external rotation and shortening of the right antebrachium; d) Craniocaudal view radiographs made immediately following the operation. Distal ulnar ostectomy and distal radial osteotomy were performed and a four ring construct with linear motor was applied; e) Craniocaudal view radiographs made 28 days following the operation. The proximal and distal block rings have been distracted away from the section of the osteotomy. Note the regenerate bone formation in the distraction gaps; f) Lateral view radiographs obtained 83 days following the operation prior to fixator removal. The regenerate has consolidated in the distraction gaps
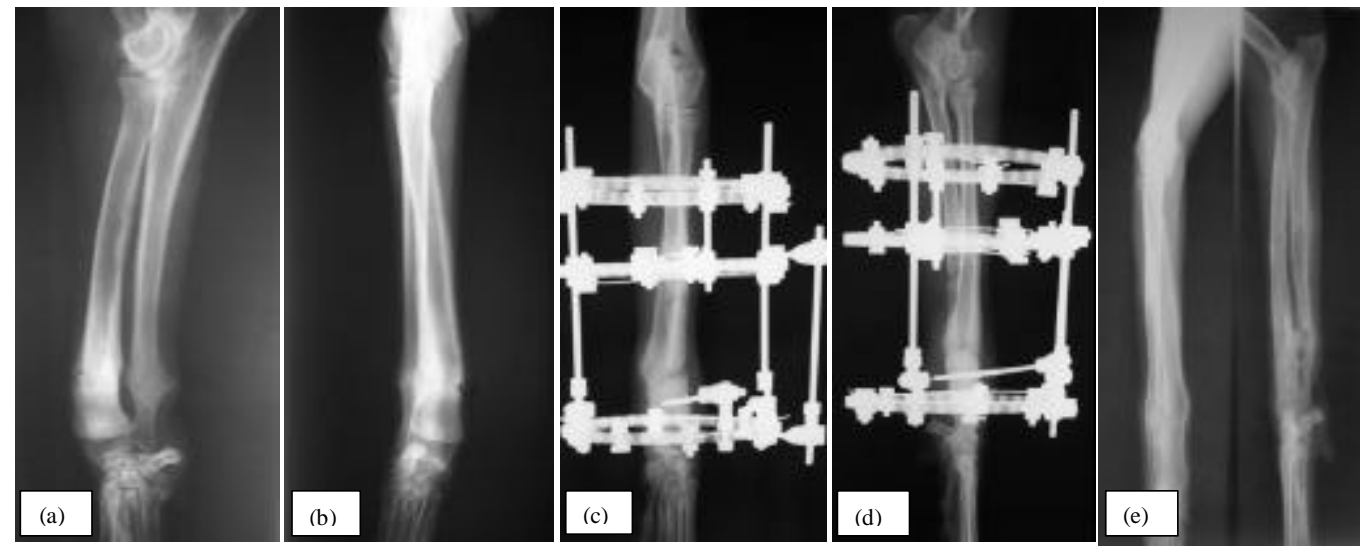

Fig. 3: a, b) Lateral and craniocaudal view radiographs of the left antebrachium of dog (No. 8). The distal ulnar physis has closed prematurely resulting in a shortened ulna; c) Craniocaudal view radiographs after correction of the deformity with the angular motor obtained 21 days following the operation; d) Lateral view radiographs of the antebrachium during the consolidation period of the bone after removing the angular motor; e) Craniocaudal and lateral view radiographs obtained 138 days following the operation. The dog had excellent limb function and there is remodeling of the regenerate bone

placed perpendicular to the plane of deformity. The rings were in a parallel alignment following correction of the angular component of the deformity in all oblique plan deformity dogs and the angular motor and hinges assemble were replaced with linear motors to perform linear distraction.

During lengthening, after angular and rotational correction of deformity in $\operatorname{dog}$ (No. 6), there was premature consolidation of the regenerate resulted in incomplate lengthening $(5 \mathrm{~mm}$ deficiency of length contrary to the right radius) of the left radius. Latency period ranged from 3-7 days (in immature dogs 3-5 days, mature dogs 5-7 days). A distraction rate of $1 \mathrm{~mm}^{\text {day }}{ }^{-1}$ was used in all dogs. Distraction was performed twice daily in all dogs. The distraction period ranged from 7-29 days. Lengthening achieved ranged from $7-25 \mathrm{~mm}$ of the bone's initial length. Minor wire track inflamation occured in eight dogs with three dogs (No. 2, 4 and 9) also having wire track infection. No post-operative neurological impairment was observed in any dogs. Flexure contracture 
of the carpus was a temporary complication in two dogs (No. 3 and 4) undergoing antebrachial lengthening of the distal antebrachium. Contracture was managed by physical therapy (Intermittent forced extension of the elbow and carpus) and the administration of nonsteroidal antiinflamatory drogs (carprofen $2.2-4.2 \mathrm{mg}$ $\mathrm{kg}^{-1}, \mathrm{PO}, \mathrm{BID}$, Rimadyl, Pfizer-Turkey). All dogs had effective regenerate bone formation within the distraction gap.

Early regenerate bone formation was characterized radiographically with an intermediate radiolucent interzone. Fixator removal ranged from 45-93 days following the operation after radiographic consolidation of the regenerated bone.

Follow-up evalution by clinical and radiological examination ranged from 95-150 days. Limb functions were assessed to be excellent in four dogs, good in four dogs and fair in two dogs. Cosmetic results were assessed to be excellent in five dogs, good in four dogs and fair in one dog.

\section{DISCUSSION}

This case series demostrates the utility of the CESF system for performing bone lengthening and correction of angular and rotational deformity. Angular deformity correction and limb lengthening have often been difficult cases for veterinary surgeons to manage because restraints imposed by the surrounding soft tissues often prevent complete acute correction of the deformities (Forell and Schwarz, 1993; Lesser, 1994; Preston, 2000; Quinn et al., 2000; Yanoff et al., 1992) however, progressive lengthenings and/or angular correction can be successfuly performed in dogs (Ferretti, 1998; Latte, 1998; Marcellin-Little et al., 1998) using the methods of Ilizarov. The surgeon must be able to accurately define the deformity and have a thorough understanding of the equipment and methods utilized to perform the correction to obtain optimal results (Ferretti, 1998; Latte, 2002; Marcellin-Little, 1999; Stallings et al., 1998).

Linear antebrachial lengthening were much simpler to plan and perform than angular and/or rotational correction. Angular and rotational deformity was observed at the same time in five (No. 3, 4, 6, 9 and 10) of these dogs. The IECF was applied before osteotomy of the principal bone being lengthened was done, maintaing stability of the bone-CF construct throughout the procedur. Correction of minor angular and rotational deformity was peformed acutly during the operation in four dogs (No. 3, 4, 6 and 9) and in the one dog (No. 10) rotational deformity was corrected acutely and totaly.
Effective correction of angular deformities is predicted on proper hinge placement (Lewis et al., 1999b; Marcellin-Little et al., 1998; Marcellin-Little, 1999). The hinges should be positioned at the apex of the deformiy (To prevent translation from occurring during distraction) and perpendicular to the plane of the deformity (to effectively correct angular deformity) (Lewis et al., 1999b). To define angular and rotational deformities, researchers used graphical and mathematical methods which proved accurate and simplified the process.

Accurate placement of the initial fixation wire, parallel to the articular surface of the distal radius and in the correct longitudinal position as pre-determined from the preoperative radiograph was critical to accurate positioning of the hinges at the apex of the deformity. Osteotomy of the radius was performed after distal ulnar ostectomy and the CF was appied. Initially, researchers corrected rotational deformity sequentially during the post operative distraction period because of concerns that acute correction of large rotational deformity at the time of the operation might result in vascular compromise (Edema) of the distal limb. Correcting the rotational component of a deformity could potentially alter the plane of deformity, however if the osteotomy is made at or preferable slightly distal to the point of maximal deformity, rotation of the distal bone segment should have a negligible effect on the plane of deformity.

Acute correction of angular and rotational deformities can result in alignment of the bone segments at the osteotomy that is less than optimal for bone healing (Lewis et al., 1999b; Marcellin-Little et al., 1998) and acute definitive correction is often limited by tension in the regional soft tissues. The hinged Ilizarov ESF allowed precise, progresive gradual lengthening and correction of angular deformities.

Progressive lengthening and angular corrections were initiated after a 3-7 days latency. Latency is postulated to improve osteogenesis by allowing the formation of a fibrovascular bridge which serves as a framework for intramembranous ossification during controlled distraction. Immediate distraction may have detrimental effects on osteogenesis occurring in the distraction gap by inhibiting the repair of damaged blood vessels and the proliferation of osteogenic precursor cells (Ilizarov, 1989a). Ilizarov (1989b)'s clinical experiences and experimental observations led him to recommend a 5-7 days latency before commencing distraction. Although, the value of a latency period has been questioned, experimental investigations evaluating osteotomies in rabbits subjected to immediate distraction had inferior regenerate bone formation compared to 
osteotomies distracted after a 7 days latency period (Lewis et al., 1999b). Recent report in the veterinary literature suggest a 3-5 days latency period in dogs and cats. A 1-3 days latency is likely sufficient in skeletally immature dogs and cats. A 5-7 days delay period is recommended in all gradual lengthenings and bone transport procedures in adult dogs. However, the latency should be determined in each individual case based on the age and overall health of the dog, the bone involved, the location of the osteotomy and degree of associated soft tissue trauma (Stallings et al., 1998). In the study, dogs up to 12 months old had a latency period of 3-5 days and adult dogs had 5-7 days. Researchers observed that there was enough intramembranous ossification in the distraction area.

In Ilizarov's experiments, optimal regenerate bone formation was optained with a rate of $1 \mathrm{~mm}$ day $^{-1}$ distracted in 60 equal increments $(0.017 \mathrm{~mm}$ every $24 \mathrm{~min})$ controlled by a mechanical autodistractor (Lewis et al., 1999b; Stallings et al., 1998). However, a distraction rate of $1.0 \mathrm{~mm}$ day $^{-1}$ deviced into four equal increments $(0.25 \mathrm{~mm}$ every $6 \mathrm{~h})$ was advised as the most practical protocol for producing quality regenerate bone with minimal morbidity (Ilizarov, 1989b; Stallings et al., 1998). At a distraction rate of $1 \mathrm{~mm}$ day $^{-1}$ in one step every $24 \mathrm{~h}$, the fascia became stretched in appearance and many areas of homogenization, indicating structural damage were noted. Similarly, rhythm increments can be altered to two or three daily increments especially related with deficiency of the original bone's length (Ilizarov, 1989b). Distraction rates can be increased if the regenerate appears to be consolidating prematurely and decreased if the regenerate bone formation is poor or if soft tissue contraction is marked (Lewis et al., 1999b). Complications such as premature consolidation, neuropraxia, muscle and tendon contractures, joint subluxation and poor regenerate formation are more likely to occure if substantial alterations of the established rate and rhythm guidelines are used (Lewis et al. 1999b; Stallings et al., 1998). A distraction rate of $1 \mathrm{~mm}$ and a twice daily rhythm was used in all of the dogs in this study and occured good regenerate bone formation. An accelerated distraction rate to $1.5 \mathrm{~mm}$ a day divided into 2 equal increments was used temporary in dogs (No. 3 and 4) but the accelerated rate aggravated flexure contracture in this dogs. The rate was subsequently decreased to $1 \mathrm{~mm}$ a day. One skeletally immature dog (No. 6) had premature consolidation of the regenerate in the distraction period. Premature consolidation terminated further distraction in this dog. For this reason, compared to the other leg a $5 \mathrm{~mm}$ shortening remained.
The daily distraction of $1 \mathrm{~mm}$ was performed with the help of nuts from the Ilizarov system rods. On the square-shaped nuts figures from 1-4 are available and each $1 / 4$ round of the nut, ensures a bone distraction of $0.25 \mathrm{~mm}$. Distraction was performed simply and precisely without any apparent discomfort and was done in all cases by the dogs' owner at home. While distraction was being performed, the patients were recall to the clinic every weeks to recheck and assess the progress of distraction.

The most common complication in this case series was wire track inflammation (Lewis et al., 1999a; Marcellin-Little et al., 1998). This is linked to length of stay of the fixation and the muscle mass volume. Although, the post operative dressings of the extremities were very well done, researchers still encountered with wire track infections, particularly in the proximal bone fragments. Researchers belive that the reason for this stems from having more muscle mass in the region. Flexure contracture was also a problem in dogs undergoing angullar correction and/or lengthening of the distal antebrachium. Physical therapy, intermittent coaptation in forced extension and administration of anti-inflatatory or analgesic drags seemed to relieve the magnitute of contracture. Contracture was temporary in some dogs and range of motion in the antebrachiocarpal joint had improved substantially by the time of fixator removal.

\section{CONCLUSION}

Except two cases (No. 2 and 6), the outcome was good to excellent in this study. These two dogs not losing their lameness was the result of not been able to fully suppres the elbow incongruity. Residual deformities were small in all cases treated in this study. Efforts in the future should be directed to mitigate these problems and improve joint and limb function in association with musculoskeletal deformity correction.

\section{REFERENCES}

Ferretti, A., 1998. Fracture, nonunion and deformity treatment with the ilizarov method. Proceedings of the 4th European FECAVA/ SCIVAC Congress, June 18-21, 1998, Bolognia, pp: 471-475.

Forell, E.B. and P.D. Schwarz, 1993. Use of external skeletal fixation for treatment of angular deformity secondary to premature distal ulnar physeal closure. J. Am. Anim. Hospital Assoc., 29: $460-476$. 
Fox, S.M., J.C. Bray, S.R. Guerin and H.M. Burbridge, 1995. Antebrachial deformities in the dog: Treatment with external fixation. J. Small Anim. Pract., 36: 315-320.

Ilizarov, G.A., 1989a. The tension-stress effect on the genesis and growth of tissues: Part I. The influence of stability of fixation and soft tissue preservation. Clin. Orthop. Rel. Res., 238: $249-281$.

Ilizarov, G.A., 1989b. The tension stress effect on the genesis and growth of tissues: Part II. The influence of the rate and frequency of distraction. Clin. Orthop. Related Res., 239: 263-285.

Latte, Y., 1998. A review of 75 applications of the Ilizarov method. Part 2. Eur. J. Companion Anim. Pract., 8: 64-81.

Latte, Y., 2002. Management of axial deformities by circular fixators. Proceedings of the 1st World Orthopaedic Veterinary Congress and 11th Annual ESVOT Congress, September 5-8, 2002, Munich, Germany, pp: 120-122.

Lesser, A.S., 1994. Segmental bone transport for the treatment of bone deficits. J. Am. Anim. Hospital Assoc., 30: 322-330.

Lesser, A.S., 1998. Ilizarov Technique. In: Current Techniques in Small Animal, Bojrab, M.J. (Ed.). 4th Edn. Williams and Wilkins, Baltimore, MA., USA., pp: $950-963$.

Lewis, D.D., R.M. Radasch, B.S. Beale, J.T. Stallings, O.I. Lanz, R.D. Welch and M.L. Samchukov, 1999a. Initial clinical experience with the $\mathrm{IMEX}^{\mathrm{TM}}$ clinical external skeletal fixation system. Part I: Use in fractures and arthrodeses. Vet. Comp. Orthop. Traumatol., 12: 108-117.
Lewis, D.D., R.M. Radasch, B.S. Beale, J.T. Stallings, R.D. Welch, M.L. Samchukov and O. Lanz, 1999b. Initial clinical experience with the IMEX ${ }^{\mathrm{TM}}$ circular external skeletal fixation system. Part II: Use in bone lengthening and correction of angular and rotational deformities. Vet. Comp. Orthop. Traumatol., 12: 118-127.

Marcellin-Little, D.J., 1999. Treating bone deformities with circular external skeletal fixation. Compendium Continu. Educ. Pract. Vet., 21: 481-486.

Marcellin-Little, D.J., A. Ferretti, S.C. Roe and D.J. De Young, 1998. Hinged Ilizarov external fixation for correction of antebrachial deformities. Vet. Surg., 27: $231-245$.

Preston, C.A., 2000. Distraction osteogenesis to treat premature distal radial growth plate closure in a dog. Aust. Vet. J., 78: 387-391.

Quinn, M.K., N. Ehrhart, A.L. Johnson and D.J. Schaeffer, 2000. Realignment of the radius in canine antebrachial growth deformities treated with corrective osteotomy and bilateral (Type II) external fixation. Vet. Surg., 29: 558-563.

Stallings, J.T., D.D. Lewis, R.D. Welch, M. Samchukov and D.J. Marcellin-Little, 1998. An introduction to distraction osteogenesis and the principles of the ilizarov method. Vet. Comp. Orthop. Traumatol., 11: 59-67.

Yanoff, S.R., D.A. Hulse, R.H. Palmer and M.R. Herron, 1992. Distraction osteogenesis using modified external fixation devices in five dogs. Vet. Surg., 21: $480-487$. 